\title{
Predictors of Lymphoceles in Women Who Underwent Laparotomic Retroperitoneal Lymph Node Dissection for Early Gynecologic Cancer: A Retrospective Cohort Study
}

\author{
Hui-Hua Chen ${ }^{1,+}+$, , Wan-Hua Ting ${ }^{1,+}$, Ho-Hsiung Lin ${ }^{1,2}$ and Sheng-Mou Hsiao ${ }^{1,2,3, * \mathbb{C}}$ \\ 1 Department of Obstetrics and Gynecology, Far Eastern Memorial Hospital, Banqiao, New Taipei 220, Taiwan; \\ thandaaye24@gmail.com (H.-H.C.); stellatingwh@yahoo.com (W.-H.T.); hhlin@ntuh.gov.tw (H.-H.L.) \\ 2 Department of Obstetrics and Gynecology, National Taiwan University College of Medicine and the \\ Hospital, Taipei 100, Taiwan \\ 3 Graduate School of Biotechnology and Bioengineering, Yuan Ze University, Taoyuan 320, Taiwan \\ * Correspondence: smhsiao2@gmail.com; Tel.: +886-8966-7000 (ext. 1424); Fax: +886-8966-5567 \\ + These authors contributed equally to this work.
}

Received: 27 December 2018; Accepted: 12 March 2019; Published: 15 March 2019

\begin{abstract}
Background: Lymphoceles could represent a detrimental complication after retroperitoneal lymph node dissection. Our aim was to elucidate predictors of lymphoceles. Methods: Between 2011 and 2017, medical records of consecutive women who underwent laparotomic retroperitoneal lymph node dissection for FIGO stage I or II gynecologic cancer were reviewed. Results: A total of 204 women, including those with lymphoceles $(n=31)$ and symptomatic lymphoceles $(n=7)$, were reviewed. According to multivariable analysis, parity (odds ratio $=0.59, p=0.003$ ), adjuvant pelvic radiotherapy (odds ratio $=2.60, p=0.039$ ), and peritoneal nonclosure without pelvic drainage (odds ratio $=2.31, p=0.048$ ) were predictors of lymphoceles. In addition, parity (odds ratio $=0.73, p=0.03$ ), hypertension (odds ratio $=2.62, p=0.02$ ), and peritoneal partial closure with pelvic drainage (odds ratio $=0.27, p=0.02$ ) were predictors of complications. Conclusion: Low parity, adjuvant pelvic radiotherapy, and peritoneal nonclosure without pelvic drainage were associated with increased lymphocele formation. In addition, a lower complication rate was found in the peritoneal partial closure with pelvic drainage group; thus, peritoneal partial closure with pelvic drainage might be suggested for women who undergo laparotomic retroperitoneal lymph node dissection.
\end{abstract}

Keywords: lymph node excision; lymphocele; peritoneum; drainage; gynecology

\section{Introduction}

Retroperitoneal lymph node dissection is included in the treatment of oncogynecological operative procedure. One of the common complications of retroperitoneal lymph node dissection is the formation of lymphoceles.

Retroperitoneal lymph node dissection would result in disruption of lymphatic tracts and leakage of lymphatic fluid. Lymphoceles are lymphatic fluid collections in retroperitoneal space and were first described in 1955 [1]. The incidence of lymphocele formation after retroperitoneal lymph node dissection ranges from 1 to $58 \%$ [1-5]. Most lymphoceles are asymptomatic. They may resolve spontaneously with the development of new lymphatic vessels [6]. However, 5 to $34.5 \%$ of lymphoceles are symptomatic [5,7-10]. Symptoms of lymphoceles are usually related to compression to adjacent organs, infection, or fistula. Symptomatic lymphoceles usually need to be treated, and may 
result in a delay in postoperative adjuvant therapy. Thus, it is important to minimize the incidence of lymphoceles.

Following pelvic lymph node dissection, different surgical techniques have been suggested to prevent surgery-related morbidity, such as peritoneal nonclosure and implantation of a retroperitoneal suction drainage catheter.

Peritoneal closure has been recommended after pelvic and/or para-aortic lymph node dissection; however, more recently, several studies have shown that peritoneal closure could elevate the risk of lymphocele formation [9-11]. It has been suggested that the peritoneum should be left open to allow the lymphatic fluid flows into the peritoneal cavity and be absorbed [12,13]. However, some studies did not find increased lymphoceles after peritoneal closure compared with nonclosure $[14,15]$.

In addition, pelvic retroperitoneal drainage catheter insertion is recommended to prevent lymphocele formation [16]. However, Charoenkwan et al. reported that tube drain placement is associated with a higher risk of short- and long-term symptomatic lymphocele formation [17]. Franchi et al. reported that there was no difference in the lymphocele rate between the drainage and nondrainage groups [18]. We were interested in which procedures would decrease lymphocele formation.

Besides, systemic lymphadenectomy did not improve overall survival in patients with optimally debulked advanced ovarian cancer [19], and we did not routinely perform systemic lymphadenectomy in patients with advanced ovarian cancer. Therefore, only patients with early gynecologic cancer were enrolled in this study. Thus, the aim of this study was to evaluate predictors of lymphoceles in early gynecologic cancer patients who underwent laparotomic retroperitoneal lymph node dissection.

\section{Materials and Methods}

The medical records of women with a Federation of Gynecologic and Obstetrics (FIGO) stage I or II gynecological cancer, including cervical cancer, endometrial cancer, ovarian cancer, and fallopian tubal cancer, who underwent laparotomic retroperitoneal lymph node dissection from January 2011 to December 2017 in Far Eastern Memorial Hospital, a tertiary referral center, were reviewed. Far Eastern Memorial Hospital Research Ethics Review Committee approved the protocol (No: FEMH-107150-E; ClinicalTrials.gov Identifier: NCT03765320, date of registration: 5December2018).

Retroperitoneal lymph node dissection was defined as the removal of lymphatic tissue in the pelvic regions with or without para-aortic lymph node dissection. The choice of peritoneal closure methods, including complete closure, partial closure (i.e., the peritoneum was closed by interrupted sutures with open space $\sim 3 \mathrm{~cm}$ between sutures), and nonclosure, was based on each surgeon's preference. The decision of retroperitoneal suction drainage was also dependent on each surgeon's preference. De novo abdominal or pelvic cysts in ultrasonography, computed tomography, or magnetic resonance imaging after para-aortic and/or pelvic lymphadenectomy were considered to have lymphoceles. Additionally, perioperative complications, adjuvant chemotherapy, adjuvant radiotherapy, and clinical oncologic outcome were also reviewed. Parity was determined by the numbers of pregnancies reaching 20 weeks and not by the number of fetuses delivered [20].

The STATA software program (Version 11.0; Stata Corp, College Station, TX, USA) was used for statistical analyses. Chi-square test, Fisher's exact test, Wilcoxon rank-sum test, or Spearman's rank-correlation coefficient were employed for statistical analysis. Multivariable backward stepwise analysis was performed using all variables in the univariate analysis until all remaining variables became significant. A $p$-value of less than 0.05 was considered statistically significant.

\section{Results}

Two-hundred-and-four women who underwent laparotomic surgery and retroperitoneal lymph node dissection for early gynecologic cancers (i.e., stage I or II gynecologic cancer) were included in this study. The baseline data of the women are listed in Table 1. All patients underwent pelvic lymph node dissection, and 127 (62.3\%) patients underwent concomitant para-aortic lymph node 
removal. All patients in the peritoneal complete closure group $(n=44)$ and 44 of 45 patients in the peritoneal partial closure group received concomitant pelvic drainage. Nonetheless, only 24 patients in the peritoneal nonclosure group $(n=115)$ received concomitant pelvic drainage.

Table 1. Baseline data of women who underwent laparotomic surgery for early gynecologic cancers $(\mathrm{n}=204)$.

\begin{tabular}{cc}
\hline Variables & Values \\
Age (years) & $53.3 \pm 10.6$ \\
Parity & $1.9 \pm 1.4$ \\
Body mass index $\left(\mathrm{kg} / \mathrm{m}^{2}\right)$ & $26.0 \pm 5.8$ \\
Ovarian cancer or borderline tumor $(\mathrm{stage} \mathrm{I}, \mathrm{n}=56$; stage 2, $\mathrm{n}=13)$ & $69(34)$ \\
Endometrial cancer (stage I, $\mathrm{n}=84 ;$ stage 2, $\mathrm{n}=7)$ & $91(45)$ \\
Cervical or vaginal cancer (stage I, $\mathrm{n}=41$; stage 2, $\mathrm{n}=3)$ & $44(22)$ \\
Diabetes & $24(12)$ \\
Hypertension & $56(27)$ \\
Peritoneal complete closure with pelvic drainage & $44(22)$ \\
Peritoneal partial closure with pelvic drainage & $44(22)$ \\
Peritoneal partial closure without pelvic drainage & $1(1)$ \\
Peritoneal nonclosure with pelvic drainage & $24(12)$ \\
Peritoneal nonclosure without pelvic drainage & $91(45)$ \\
Pelvic drainage & $112(55)$ \\
Adjuvant pelvic radiotherapy & $43(21)$ \\
Adjuvant vaginal brachytherapy & $31(15)$ \\
Adjuvant chemotherapy & $75(34)$ \\
Complications & $50(12)$ \\
Lymphocyst & $31(14.7)$ \\
Symptomatic lymphocyst & $7(3.4)$ \\
Postoperative ileus & $9(4.4)$ \\
Miscellaneous & $11(5.3)$ \\
Recurrence of cancer & $18(8.8)$ \\
\hline
\end{tabular}

Data were expressed as the mean \pm standard deviation or number (percentage).

Among the 204 women, thirty-one (15.1\%) patients had lymphoceles. Symptomatic lymphoceles, including compression symptoms $(n=4)$ and infection $(n=3)$, were found in seven women. A variety of treatments, including computerized tomographic guided drainage $(n=4)$, laparoscopic marsupialization $(n=1)$, aspiration $(n=1)$, and intravenous antibiotics $(n=1)$, were used to treat patients with symptomatic lymphoceles.

Comparisons of the incidences of lymphoceles and complications were tabulated in Table 2. There were significant between-group differences in the body mass index and the complication rates (Table 2). Nonetheless, multivariable backward stepwise logistic analysis was performed using all variables in the univariate analysis to predict lymphoceles. Parity (odds ratio $=0.59, p=0.003$ ), adjuvant pelvic radiotherapy (odds ratio $=2.60, p=0.039$ ), and peritoneal nonclosure without pelvic drainage (odds ratio $=2.31, p=0.048$ ) were predictors of lymphoceles (Table 3 ). 
Table 2. Subgroup comparisons of baseline data, rates of lymphoceles and complications $(n=204)$.

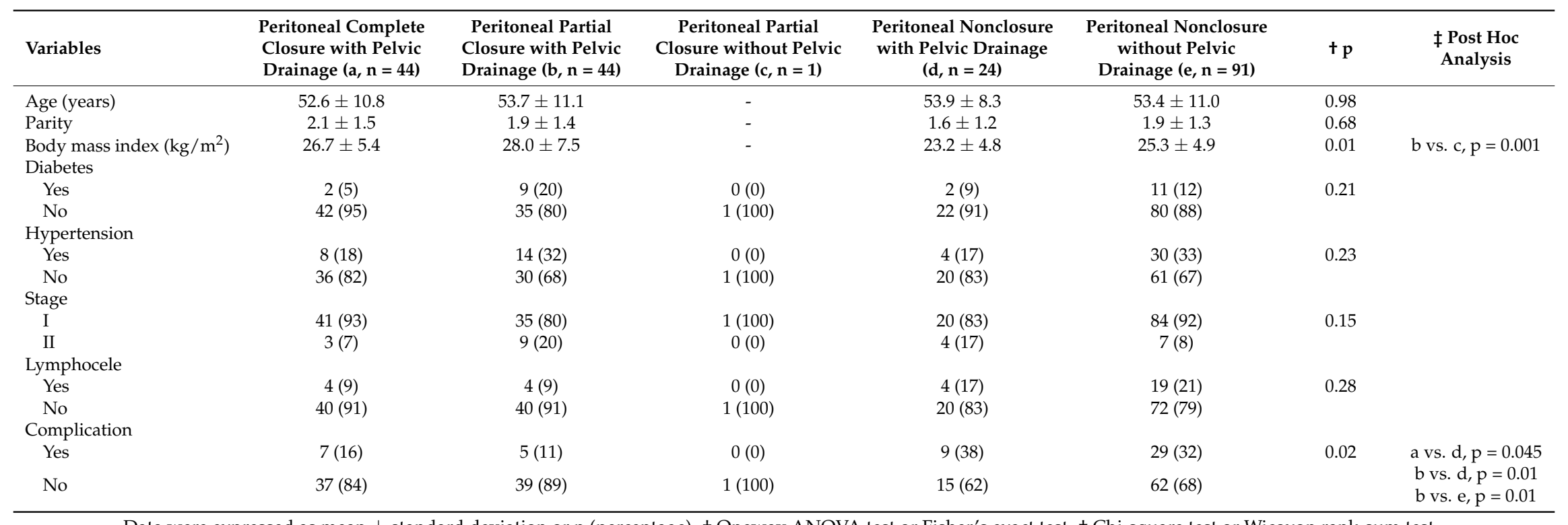

Data were expressed as mean \pm standard deviation or $\mathrm{n}$ (percentage). + Oneway ANOVA test or Fisher’s exact test. $\ddagger$ Chi-square test or Wicoxon rank-sum test. 
Table 3. Univariate and multivariable backward stepwise logistic analysis to predict lymphoceles $(\mathrm{n}=204)$.

\begin{tabular}{|c|c|c|c|c|}
\hline Variables & $\frac{\text { Univariate Analysis }}{\text { Odds Ratio }(95 \% \mathrm{CI})}$ & ${ }^{\dagger} p$ & $\frac{\text { Multivariable Analysis }}{\text { Odds Ratio }(95 \% \text { CI) }}$ & $\ddagger p$ \\
\hline Age (years) & $0.99(0.95-1.02)$ & 0.53 & - & - \\
\hline Parity & $0.62(0.45-0.86)$ & 0.004 & $0.59(0.42-0.84)$ & 0.003 \\
\hline Body mass index $\left(\mathrm{kg} / \mathrm{m}^{2}\right)$ & $0.97(0.91-1.05)$ & 0.48 & - & - \\
\hline Diabetes & $1.13(0.36-3.58)$ & 0.83 & - & - \\
\hline Hypertension & $1.10(0.47-2.55)$ & 0.83 & - & - \\
\hline Ovarian cancer & $1.09(0.49-2.43)$ & 0.83 & - & - \\
\hline Radical hysterectomy & $0.82(0.32-2.15)$ & 0.69 & - & - \\
\hline Stage of cancer & $0.82(0.23-2.94)$ & 0.76 & - & - \\
\hline Adjuvant chemotherapy & $1.77(0.82-3.82)$ & 0.15 & - & - \\
\hline Adjuvant pelvic radiotherapy & $2.42(1.06-5.56)$ & 0.04 & $2.60(1.05-6.45)$ & 0.039 \\
\hline Adjuvant vaginal brachytherapy & $1.42(0.53-3.81)$ & 0.49 & - & - \\
\hline Peritoneal complete closure with pelvic drainage $(n=44)$ & $0.49(0.16-1.49)$ & 0.21 & - & - \\
\hline Peritoneal partial closure with pelvic drainage $(n=44)$ & $0.49(0.16-1.48)$ & 0.21 & - & - \\
\hline$\S$ Peritoneal partial closure without pelvic drainage $(n=1)$ & - & - & - & - \\
\hline Peritoneal nonclosure with pelvic drainage $(\mathrm{n}=24)$ & $1.13(0.36-3.58)$ & 0.83 & - & - \\
\hline Peritoneal nonclosure without pelvic drainage $(n=91)$ & $2.22(1.01-4.86)$ & 0.046 & $2.31(1.01-5.30)$ & 0.048 \\
\hline
\end{tabular}

Data were expressed as odds ratios (95\% confidence intervals). $\mathrm{CI}=$ confidence interval. ${ }^{\dagger}$ Logistic regression analysis. ${ }^{\ddagger}$ Multivariate backward stepwise logistic analysis was performed using all variables in the univariate analysis until all remaining variables became significant. Pseudo $\mathrm{R}^{2}=0.10$. $\$$ Peritoneal partial closure without pelvic drainage predict no complication perfectly, thus logistic regression analysis cannot be performed for this variable. 
The optimum cut-off value of parity $\leq 2$ to predict lymphoceles was determined using receiver operating characteristic (ROC) curve analysis, which has an area under the ROC curve of 0.67 ( $95 \%$ confidence interval $(\mathrm{CI})=0.56$ to 0.78 ; sensitivity $=70.4 \%$, specificity $=60.0 \%$, Figure 1 ).

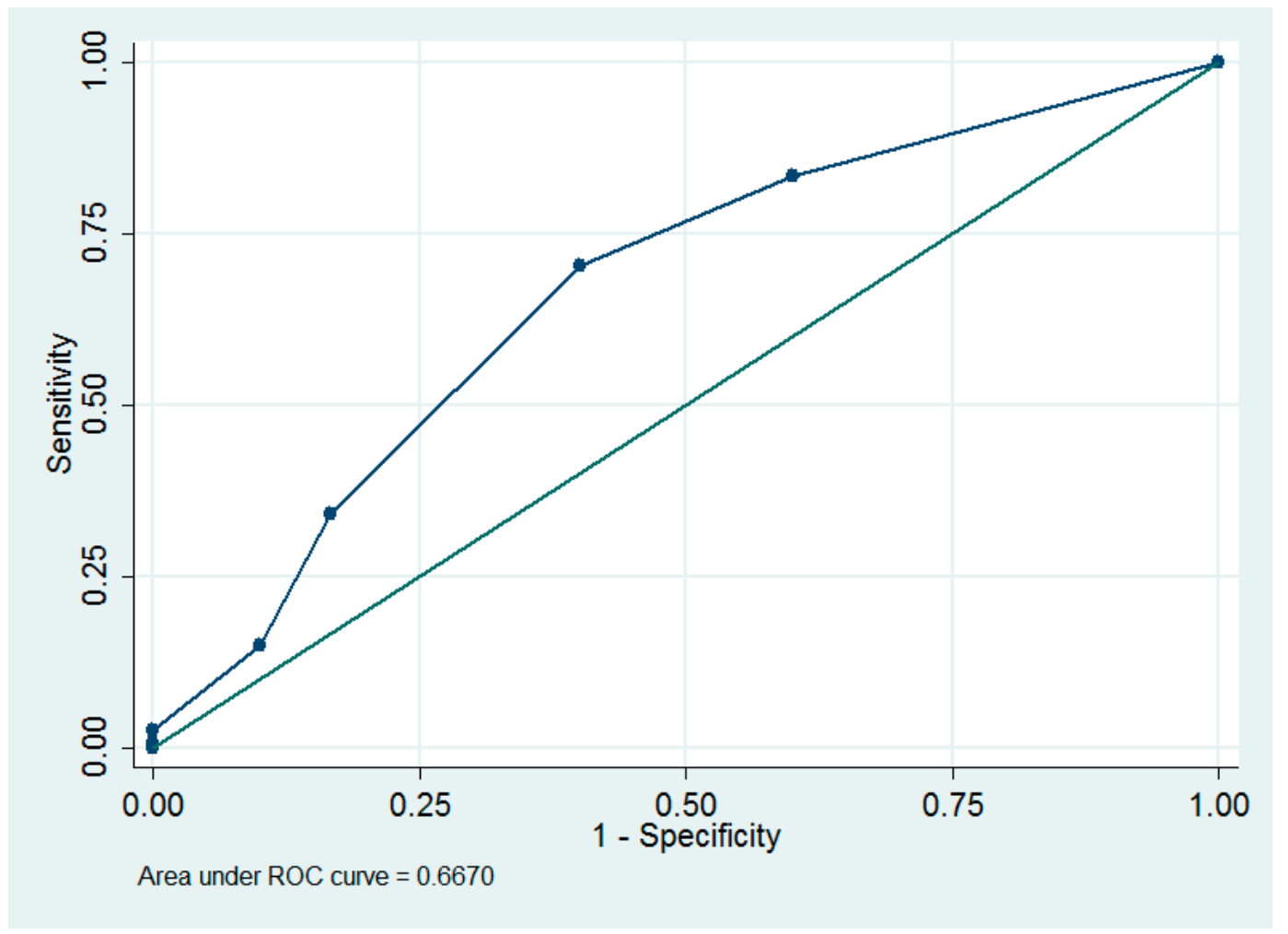

Figure 1. The receiver operating characteristic (ROC) curves of using parity to predict lymphoceles.

Complications included lymphoceles, postoperative ileus, and miscellaneous (Table 1). Multivariable backward stepwise logistic analysis was performed using all variables in the univariate analysis to predict complications. Parity (odds ratio $=0.73, p=0.03$ ), hypertension (odds ratio $=2.62$, $p=0.02$ ), and peritoneal partial closure with pelvic drainage (odds ratio $=0.27, p=0.02$ ) were predictors of complications (Table 4). 
Table 4. Univariate and multivariable backward stepwise logistic analysis to predict complications $(\mathrm{n}=204)$.

\begin{tabular}{|c|c|c|c|c|}
\hline Variables & $\frac{\text { Univariate Analysis }}{\text { Odds Ratio }(95 \% \text { CI) }}$ & ${ }^{\dagger} p$ & $\frac{\text { Multivariable Analysis }}{\text { Odds Ratio }(95 \% \mathrm{CI})}$ & $\ddagger p$ \\
\hline Age (years) & $1.02(0.99-1.05)$ & 0.19 & - & - \\
\hline Parity & $0.82(0.63-1.05)$ & 0.11 & $0.73(0.55-0.96)$ & 0.03 \\
\hline Body mass index $\left(\mathrm{kg} / \mathrm{m}^{2}\right)$ & $0.97(0.91-1.03)$ & 0.25 & - & - \\
\hline Diabetes & $0.79(0.28-2.24)$ & 0.66 & - & - \\
\hline Hypertension & $1.94(0.98-3.83)$ & 0.057 & $2.62(1.18-5.81)$ & 0.02 \\
\hline Ovarian cancer & $1.14(0.58-2.22)$ & 0.71 & - & - \\
\hline Radical hysterectomy & $1.34(0.64-2.81)$ & 0.44 & - & - \\
\hline Stage of cancer & $1.40(0.54-3.64)$ & 0.49 & - & - \\
\hline Adjuvant chemotherapy & $1.07(0.56-2.07)$ & 0.84 & - & - \\
\hline Adjuvant pelvic radiotherapy & $1.45(0.69-3.07)$ & 0.33 & - & - \\
\hline Adjuvant vaginal brachytherapy & $0.88(0.36-2.19)$ & 0.79 & - & - \\
\hline Peritoneal complete closure with pelvic drainage $(n=44)$ & $0.51(0.21-1.24)$ & 0.14 & - & - \\
\hline Peritoneal partial closure with pelvic drainage $(n=44)$ & $0.32(0.12-0.88)$ & 0.03 & $0.27(0.09-0.83)$ & 0.02 \\
\hline$\S$ Peritoneal partial closure without pelvic drainage $(n=1)$ & - & - & - & - \\
\hline Peritoneal nonclosure with pelvic drainage $(\mathrm{n}=24)$ & $2.03(0.83-4.99)$ & 0.12 & - & - \\
\hline Peritoneal nonclosure without pelvic drainage $(\mathrm{n}=91)$ & $2.05(1.07-3.91)$ & 0.03 & - & - \\
\hline
\end{tabular}

Data were expressed as odds ratios (95\% confidence intervals). CI = confidence interval. ${ }^{\dagger}$ Logistic regression analysis. ${ }^{\ddagger}$ Multivariate backward stepwise logistic analysis was performed using all variables in the univariate analysis until all remaining variables became significant. Pseudo $\mathrm{R}^{2}=0.07$. ${ }^{\S}$ Peritoneal partial closure without pelvic drainage predict no complication perfectly, thus logistic regression analysis cannot be performed for this variable. 
The optimum cut-off value of parity $\leq 2$ to predict complications was determined using ROC curve analysis, which has an area under the ROC curve of 0.58 (95\% CI $=0.48$ to 0.67 ; sensitivity $=69.0 \%$, specificity $=44.7 \%$, Figure 2 ).

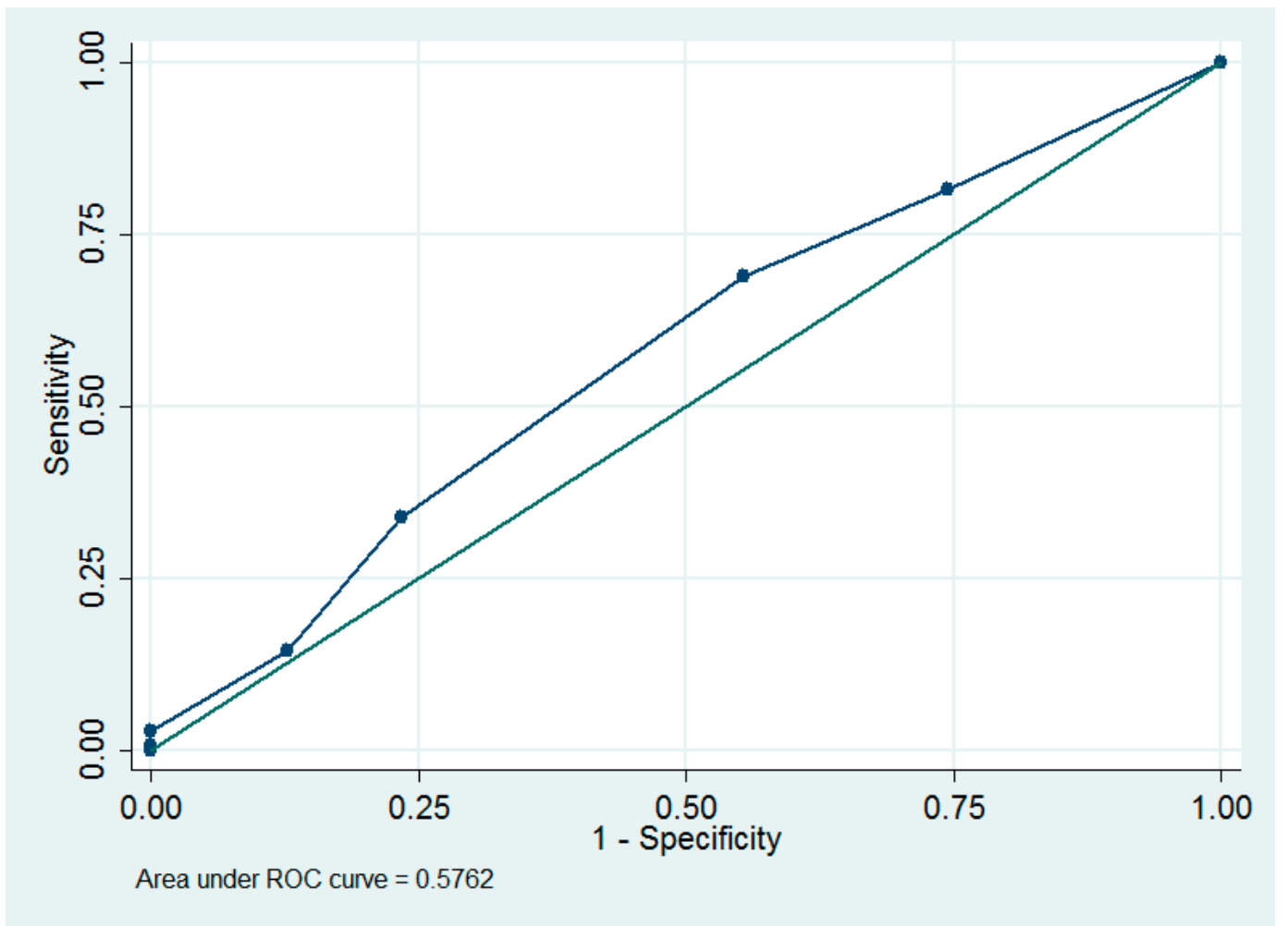

Figure 2. The receiver operating characteristic (ROC) curves of using parity to predict complications.

In addition, cancer recurrence was not associated with peritoneal partial closure with pelvic drainage, pelvic drainage, or presence of lymphoceles (all $p>0.05$, Table 5).

Table 5. Correlation of cancer recurrence and peritoneal closure/drainage/lymphoceles $(n=204)$.

\begin{tabular}{ccc}
\hline Variables & Spearman's Rho & ${ }^{\dagger} \boldsymbol{p}$ \\
\hline Peritoneal partial closure with & -0.08 & 0.26 \\
pelvic drainage & -0.10 & 0.15 \\
Pelvic drainage & -0.04 & 0.62 \\
Presence of lymphoceles &
\end{tabular}

\section{Discussion}

In our study, peritoneal nonclosure without pelvic drainage was associated with increased lymphocele formation (odds ratio $=2.31, p=0.048$, Table 3). Similarly, Bafna et al. found that peritoneal partial closure without pelvic drainage was associated with increased lymphocele formation $(28 \%)$ [15]. In contrast, some authors observed a lower incidence of lymphoceles in the peritoneal nonclosure/partial closure group compared with the closure group [12,13,21,22]. In addition, some studies reported that the incidence of lymphoceles did not differ between the peritoneal nonclosure groups and the peritoneal closure group [14,15]. Thus, whether peritoneal nonclosure could increase or decrease lymphocele formation remains undetermined.

Retroperitoneal drainage with catheter insertion is traditionally recommended to remove leaking lymph and thus prevent lymphocele formation [11]. We also found that peritoneal nonclosure without 
pelvic drainage was associated with increased lymphocele formation (odds ratio $=2.31, p=0.048$, Table 3). Nonetheless, in the Cochrane review, retroperitoneal drainage did not have benefit for preventing lymphocele formation [17], and peritoneal nonclosure with pelvic drainage was associated with a higher risk of symptomatic lymphocele formation [17]. Franchi et al. and Lopes et al. also reported that there was no difference in the incidence of lymphocele formation between the drainage and nondrainage groups $[18,23]$. However, from our study, peritoneal nonclosure with pelvic drainage (odds ratio $=1.13, p=0.83$, Table 3 ) was not a predictor for lymphocele formation; thus, it might be better to implant a pelvic drainage tube if the peritoneum is not closed.

In our study, adjuvant pelvic radiotherapy were associated with increased lymphocele formation (odds ratio $=2.60, p=0.039$, Table 3). Petru et al. also found a greater incidence of lymphoceles in cervical cancer patients who underwent postoperative adjuvant radiotherapy and/or chemotherapy compared with no adjuvant therapy ( $60 \%$ vs. $32.6 \%$, respectively, $p=0.01$ ) [9], and a more advanced disease might explain the finding of a greater incidence of lymphoceles in the patients receiving adjuvant therapy [9]. However, in our study, we included cancer stage as one of the variables in multivariable backward stepwise logistic analysis (Table 3); thus, adjuvant radiotherapy as a predictor for lymphocele formation should be less likely related to more advanced disease. Instead, we speculate that adjuvant pelvic radiotherapy might be detrimental to the development of new lymphatic vessels, thus subsequently resulting in delayed absorption of lymph and a greater incidence of lymphocele formation found during the follow-up.

In our study, increased parity was associated with decreased lymphocele formation (odds ratio $=0.59, p=0.003$, Table 3). It was reported that the number of parity was significantly correlated with microvessel counts, and this might be due to the effects of hormone stimulation during previous pregnancies [24]. Thus, we speculate that parity might be also positively correlated with lymphatic microvessels, and this may explain the positive correlation between increased parity and decreased lymphocele formation. In our study, we found that parity $\leq 2$ was an optimum cut-off value to predict lymphoceles and complications. We can use the above cut-off value as a guideline for pretreatment consultation. However, owing to their low areas under the ROC curve (Figure 1, Figure 2), parity is not a good predictor of lymphoceles and complications.

Franchi et al. reported that the infection and adhesion complication rates did not differ between the peritoneal closure and nonclosure groups [14]. However, in our study, peritoneal partial closure with pelvic drainage was associated with a lower complication rate (odds ratio $=0.27, p=0.02$, Table 4). Thus, partial peritoneal closure with pelvic drainage might be suggested for women who underwent retroperitoneal lymph node dissection. Zikan et al. reported that radical hysterectomy is an independent predictor for symptomatic lymphoceles (odds ratio $=2.208,95 \% \mathrm{CI}=1.058$ to $4.606, p=0.035$ ) [25]. In Franchi et al.'s study, all patients received either peritoneal complete closure or nonclosure, and underwent radical hysterectomy and pelvic drainage [14]. However, 45 (22\%) of our patients underwent peritoneal partial closure, 45 (22\%) of our patients underwent radical hysterectomy, and $112(55 \%)$ of our patients underwent pelvic drainage (Table 1$)$. Thus, the difference in the peritoneal closure, patient composition, and pelvic drainage between our and Franchi et al.'s studies might partly explain the between-group difference in the finding of peritoneal partial closure with pelvic drainage as a predictor of low complication rate.

In this study, hypertension was a predictor for complications (odds ratio $=2.62, p=0.02$, Table 4 ). Despite of statistical insignificance, our patients with hypertension had higher rates of postoperative ileus $(3 / 56$ vs. $6 / 148, p=0.71)$ and surgical wound infection/dehiscence $(3 / 56$ vs. $2 / 148, p=0.13)$, compared with patients without hypertension. Similarly, Sheyn et al. reported that a higher incidence of hypertension was found in patients with small bowel obstruction, compared with patients without small bowel obstruction (37.1\% vs. $27.3 \%, p<0.001)$ [26]. Kaneko et al. reported that hypertension was a risk factor for incisional hernia in patients underwent loop ileostomy closure (odds ratio $=2.60,95 \%$ $\mathrm{CI}=1.01$ to $6.69, p=0.046$ ) [27]. Hypertension has been found to promote abnormal wound healing 
by inducing endothelial dysfunction, aggravate the inflammation-induced hypoxia, and aggravate pathological skin fibroblast behavior [28].

Eighteen $(8.8 \%)$ patients had cancer recurrence (Table 1$)$. However, we did not find any association between lymphocele formation and cancer recurrence (Table 5). Besides, we did not find any literature that reported a significant association between lymphocele formation and cancer recurrence.

The limitations of this study include the retrospective nature, not a randomized trial, and a limited sample size. Further prospective randomized studies should be performed to confirm our findings. In addition, nearly all our patients in the peritoneal complete and partial closure groups had concomitant pelvic drainage. Thus, our conclusion should not be generalized for those who underwent pelvic peritoneal closure without pelvic drainage.

\section{Conclusions}

Low parity, adjuvant pelvic radiotherapy, and peritoneal nonclosure without pelvic drainage were associated with increased lymphocele formation. In addition, a lower complication rate was found in the peritoneal partial closure with pelvic drainage group; thus, peritoneal partial closure with pelvic drainage might be suggested for women who undergo laparotomic retroperitoneal lymph node dissection.

Author Contributions: Conceptualization, S.-M.H.; Methodology, S.-M.H.; Validation, H.-H.C., W.-H.T., H.-H.L., and S.-M.H.; Formal Analysis, S.-M.H.; Investigation, S.-M.H.; Resources, S.-M.H.; Data Curation, H.-H.C. and W.-H.T.; Writing-Original Draft Preparation, H.-H.C. and W.-H.T.; Writing-Review\& Editing, S.-M.H.; Visualization, S.-M.H.; Supervision, H.-H.L.; Project Administration, S.-M.H.; Funding Acquisition, S.-M.H.

Funding: This research received no external funding.

Conflicts of Interest: The authors declare no conflicts of interest.

\section{References}

1. Mori, N. Clinical and experimental studies on the so-called lymphocyst which develops after radical hysterectomy in cancer of the uterine cervix. J. Jpn. Obstet. Gynecol. Soc. 1955, 2, 178-203. [PubMed]

2. Logmans, A.; Kruyt, R.H.; De Bruin, H.G.; Cox, P.H.; Pillay, M.; Trimbos, J.B. Lymphedema and lymphoceles following lymphadenectomy may be prevented by omentoplasty: A pilot study. Gynecol. Oncol. 1999, 75, 323-327. [CrossRef] [PubMed]

3. Gray, M.J.; Plentl, A.A.; Taylor, H.C., Jr. The lymphocyst: A complication of pelvic lymph node dissections. Am. J. Obstet. Gynecol. 1958, 75, 1059-1062. [CrossRef]

4. Mann, W.J.; Vogel, F.; Patsner, B.; Chalas, E. Management of lymphoceles after radical gynecologic surgery. Gynecol. Oncol. 1989, 33, 248-250. [CrossRef]

5. Benedetti-Panici, P.; Maneschi, F.; Cutillo, G.; D'Andrea, G.; di Palumbo, V.S.; Conte, M.; Scambia, G.; Mancuso, S. A randomized study comparing retroperitoneal drainage with no drainage after lymphadenectomy in gynecologic malignancies. Gynecol. Oncol. 1997, 65, 478-482. [CrossRef] [PubMed]

6. Conte, M.; Benedetti-Panici, P.; Guariglia, L.; Scambia, G.; Greggi, S.; Mancuso, S. Pelvic lymphocele following radical para-aortic and pelvic lymphadenectomy for cervical carcinoma: Incidence rate and percutaneous management. Obstet. Gynecol. 1990, 76, 268-271. [PubMed]

7. Kim, H.Y.; Kim, J.W.; Kim, S.H.; Kim, Y.T.; Kim, J.H. An analysis of the risk factors and management of lymphocele after pelvic lymphadenectomy in patients with gynecologic malignancies. Cancer Res. Treat. 2004, 36, 377-383. [CrossRef]

8. Achouri, A.; Huchon, C.; Bats, A.S.; Bensaid, C.; Nos, C.; Lecuru, F. Complications of lymphadenectomy for gynecologic cancer. Eur. J. Surg. Oncol. 2013, 39, 81-86. [CrossRef]

9. Petru, E.; Tamussino, K.; Lahousen, M.; Winter, R.; Pickel, H.; Haas, J. Pelvic and paraaortic lymphoceles after radical surgery because of cervical and ovarian cancer. Am. J. Obstet. Gynecol. 1989, 161, 937-941. [CrossRef]

10. Benedet, J.L.; Turko, M.; Boyes, D.A.; Nickerson, K.G.; Bienkowska, B.T. Radical hysterectomy in the treatment of cervical cancer. Am. J. Obstet. Gynecol. 1980, 137, 254-262. [CrossRef] 
11. Achouri, A.; Huchon, C.; Bats, A.S.; Bensaid, C.; Nos, C.; Lecuru, F. Postoperative lymphoceles after lymphadenectomy for gynaecological malignancies: Preventive techniques and prospects. Eur. J. Obstet. Gynecol. Reprod. Biol. 2012, 161, 125-129. [CrossRef]

12. Pennehouat, G.; Mosseri, V.; Durand, J.C.; Hamelin, J.P.; Asselain, B.; Pilleron, J.P.; Salmon, R.J. Lymphoceles and peritonization following lymphadenectomy for cancer of the uterus. J. Gynecol. Obstet. Biol. Reprod. 1988, 17, 373-378. (In French)

13. Thome Saint Paul, M.; Bremond, A.; Rochet, Y. Absence of peritonization after pelvic cancer surgery. Results in 157 cases. J. Gynecol. Obstet. Biol. Reprod. 1991, 20,957-960. (In French)

14. Franchi, M.; Ghezzi, F.; Zanaboni, F.; Scarabelli, C.; Beretta, P.; Donadello, N. Nonclosure of peritoneum at radical abdominal hysterectomy and pelvic node dissection: A randomized study. Obstet. Gynecol. 1997, 90, 622-627. [CrossRef]

15. Bafna, U.D.; Umadevi, K.; Savitha, M. Closed suction drainage versus no drainage following pelvic lymphadenectomy for gynecological malignancies. Int. J. Gynecol. Cancer 2001, 11, 143-146. [CrossRef]

16. Symmond, R.E.; Pratt, J.H. Prevention of fistulas and lymphoceles in radical hysterectomy. Preliminary report of a new technique. Obstet. Gynecol. 1961, 17, 57-64.

17. Charoenkwan, K.; Kietpeerakool, C. Retroperitoneal drainage versus no drainage after pelvic lymphadenectomy for the prevention of lymphocyst formation in patients with gynaecological malignancies. Cochrane Database Syst. Rev. 2017, 6, CD007387.

18. Franchi, M.; Trimbos, J.B.; Zanaboni, F.; v d Velden, J.; Reed, N.; Coens, C.; Teodorovic, I.; Vergote, I. Randomised trial of drains versus no drains following radical hysterectomy and pelvic lymph node dissection: A European Organisation for Research and Treatment of Cancer-Gynaecological Cancer Group (EORTC-GCG) study in 234 patients. Eur. J. Cancer 2007, 43, 1265-1268. [CrossRef]

19. Panici, P.B.; Maggioni, A.; Hacker, N.; Landoni, F.; Ackermann, S.; Campagnutta, E.; Tamussino, K.; Winter, R.; Pellegrino, A.; Greggi, S.; et al. Systematic aortic and pelvic lymphadenectomy versus resection of bulky nodes only in optimally debulked advanced ovarian cancer: A randomized clinical trial. J. Natl. Cancer Inst. 2005, 97, 560-566. [CrossRef]

20. Cunningham, F.G.; Leveno, K.J.; Bloom, S.L.; Hauth, J.C.; Rouse, D.J.; Spong, C.Y. William Obstetrics, 23rd ed.; McGraw-Hill: New York, NY, USA, 2010; pp. 189-214.

21. Dimpfl, T.; Stumpfe, M.; Maassen, V.; Genz, T. Lymphocele and complications after pelvic/para-aortic lymph node excision in relation to closure of the peritoneum. GeburtshilfeFrauenheilkd 1994, 54, 233-236. (In German)

22. Suzuki, M.; Ohwada, M.; Sato, I. Pelvic lymphoceles following retroperitoneal lymphadenectomy: Retroperitoneal partial "no-closure" for ovarian and endometrial cancers. J. Surg. Oncol. 1998, 68, 149-152. [CrossRef]

23. Lopes, A.D.; Hall, J.R.; Monaghan, J.M. Drainage following radical hysterectomy and pelvic lymphadenectomy: Dogma or need? Obstet. Gynecol. 1995, 86, 960-963.

24. Pansrikaew, P.; Cheewakriangkrai, C.; Taweevisit, M.; Khunamornpong, S.; Siriaunkgul, S. Correlation of mast cell density, tumor angiogenesis, and clinical outcomes in patients with endometrioid endometrial cancer. Asian Pac. J. Cancer Prev. 2010, 11, 623-626.

25. Zikan, M.; Fischerova, D.; Pinkavova, I.; Slama, J.; Weinberger, V.; Dusek, L.; Cibula, D. A prospective study examining the incidence of asymptomatic and symptomatic lymphoceles following lymphadenectomy in patients with gynecological cancer. Gynecol. Oncol. 2015, 137, 291-298. [CrossRef]

26. Sheyn, D.; Bretschneider, C.E.; Mahajan, S.T.; Ridgeway, B.; Davenport, A.; Pollard, R. Incidence and risk factors of early postoperative small bowel obstruction in patients undergoing hysterectomy for benign indications. Am. J. Obstet. Gynecol. 2018, in press. [CrossRef]

27. Kaneko, T.; Funahashi, K.; Ushigome, M.; Kagami, S.; Goto, M.; Koda, T.; Nagashima, Y.; Shiokawa, H.; Koike, J. Incidence of and risk factors for incisional hernia after closure of temporary ileostomy for colorectal malignancy. Hernia 2018, in press. [CrossRef]

28. Arima, J.; Huang, C.; Rosner, B.; Akaishi, S.; Ogawa, R. Hypertension: A systemic key to understanding local keloid severity. Wound Repair Regen. 2015, 23, 213-221. [CrossRef]

(C) 2019 by the authors. Licensee MDPI, Basel, Switzerland. This article is an open access article distributed under the terms and conditions of the Creative Commons Attribution (CC BY) license (http:/ / creativecommons.org/licenses/by/4.0/). 